Rev. Elev. Med. vet. Pays trop. 1964, 17, no / (35-41).

\title{
Différents sérotypes de Salmonella isolés en République du Tchad *
}

\author{
por A. PERPEZAT, P. PERREAU, M. THOME, M. VIGIER \\ avec la collaboration de S. MILLOT \\ Institul d’Elevage et de Médecine vétérinaire des Pays tropicaux \\ (Laboraloires de Farcha-Fort-Lamy et d'Alfort)
}

\begin{abstract}
RÉSUMÉ
Les diagnostıcs de routine et les enquêtes systématiques effectuées par le Laboratoire de Farcha dans la République du Tchad depuls 10 ans, ont permis d'isoler chez les hommes et neuf espèces animales, cent seize souches de Salmonelles, apportenant à cinquante sérolypes différents dont douze sont nouveaux.
\end{abstract}

Le travall que nous présentons est la synthèse de tous les résultats obtenus depuis dix ans dans le domaine des Salmonelles par le laboratoire de Farcha au cours des diagnostics de routine tant chez l'homme que chez l'animal et au cours des enquêtes qui en ont découlé. II permet de montrer l'importance probable des Salmonelloses humaines et animales au Tchad ainsi que la diversité des sérotypes que l'on peut rencontrer.

Les premières salmonelles furent identıfiées à partır de prélèvements qui nous avaient été adressés pour analyse bactériologique par les différents secteurs vétérinaires du Tchad ainsi que par les hôpitaux de Fort-Lamy.

Les résultats obtenus nous ont incité à entreprendre des recherches systématiques qui.

$\left(^{*}\right)$ Nous remercions Irès vivement Monsieur le Professeur LE MINOR qui a bien voulu déterminer tous les sératypes des souches que nous lui avons adressées. commencées en 1958, furent poursuivies jusqu'en 1963 avec des interruptions dues à un manque de personnel.

Pour ne pas surcharger notre exposé, nous résumerons par espèce, les conditions dans lesquelles nous avons opéré et les résultats obtenus.

\section{HOMME}

Les salmonelles citées furent isolées d'hémocultures ou de coprocultures effectuées à partir de prélèvements qui nous ont été adressés par les hôpitaux de Fort-Lamy.

Nous avons déterminé :

\section{Après hémoculture (119)}

Solmonello poratyphi A............... 1

Salmonella typhi ..................... 2

Salmonella dublin ..................... 1 


\section{Après coproculture (284)}

Solmonello paratyphi C............... 1

Solmonella ugonda..................... 1

Salmonella singapore. .................... 1

Salmonella hull........................ 2

Salmonella branderup .................... 1

Solmonella colindole . . . . . . . . . . . . . . . . . . 1

Salmonella infantis..................... 2

Salmonella monhotion................... 1

Salmonella paratyphi B. var. java .......... 1

Salmonella shubra ...................... 1

Salmonella stanleyville................ 2

Salmonella typhi...................... 1

Solmonella derby ..................... 1

Salmonella ligna (nouveau sérotype).......... 1

Salmonella chagoua (nouveau sérotype)....... 2

\section{BOVINS}

Les déterminations obtenues furent essentiellement le résultat de recherches systématiques effectuées sur des ganglions mésentériques de zébus sacrifiés à l'abattoır de Fort-Lamy, pour la consommation dans cette ville et les exportations de viande.

\section{La méthode d'isolement est la suivante:}

1. - Prélèvement, effectué le plus proprement possıble, d'un ganglion mésentérique dès l'ouverture de la cavité abdominale. Mise en boîte de Petri stérile.

2. - Au laboratoire, décapsulation du ganglion avec des instruments stériles. Ensuite, découpe en petits fragments qui sont plongés dans un tube de milieu Muller Kaufmann.

3. - A partir de ce tube, et après 24 heures d'éfuve à 370 , ensemencement sur milieu de Kristensen au vert brillant ou sur 5. S. Les colonies lactose ( $)$ sont ensuite repiquées, et un premier diagnostic d'orientation est effectué sur les milieux d'identification rapide des entérobactéries préconisés par I'Institut Pasteur de Paris (5). Tous les sérotypes des différentes souches isolées ont été déterminés par le Service des Entérobactéries de Monsieur le Professeur LE MINOR.

Furent ainsi identifiées après examen de 272 ganglions mésentériques et de 41 prélèvements divers :
Salmonella enteritidis ................. 5

Salmonella dublin ..................... 2

Salmonella amager .................... 1

Salmonello bailon .................... 1

Salmonella millesi (nouveau sérotype) . . . . . . . 1

Solmonella tchad (nouveau sérotype) ......... 1

\section{CAPRINS}

La recherche systématique des Salmonelles a été effectuée dans les ganglions mésentériques soit de chevreaux inoculés de virus capripestique (pour la production de virus pestique caprinisé) et abattus en phase d'hyperthermie, soit de chèvres sacrifiées à l'abattoir de Fort-Lamy.

La technique de prélèvement ef d'identification fut la même que celle utilisée avec les ganglions mésentériques de zébu.

Sur 163 ganglions examinés, 14 souches ont été isolées :

Salmonella dublin .................... 1

Solmonella enteritidis ................... 1

Solmonello infontts ...................

Salmonella kalomu .................. 1

Salmonello thiaroye ................... 1

Salmonella teschie ..................... 1

Solmonella leuwarden .................. 1

Salmonella amager .................. 1

Solmonello omifisan $\ldots \ldots \ldots \ldots \ldots \ldots \ldots \ldots 1$

Solmonello vejle ....................... 1

Solmonello babelsberg ................... 1

Salmonella meskin (nouveau sérotype) ........ 1

Solmonella maro (nouveau sérotype)......... 1

Salmonella farcha (nouveau sérotype)....... 1

Il est à constater que tous les isolements ne furent effectués que sur les ganglions de chèvres inoculées et non sur ceux de chèvres abattues pour la consommation humane. Cela permet de supposer que le virus inoculé déclenche la sortie des salmonelles. Ce phénomène est à rapprocher des constatations faites par PLOWRIGHT en Nigéria (6) à propos de lo mortalité observée sur les veaux lors de l'immunisation par le virus pestique atténué. Les salmoneiloses de sortie paraissent responsables d'une partie de la mortalité attribuée couramment au seul virus. 


\section{PORCINS}

Sur 17 ganglions mésentériques prélevés à l'abattoir, aucun isolement ne fut positif et la seule salmonelle mise en évidence le fut à partir de la moèlle d'un os qui nous avait été envoyé pour diagnostic de routine, dans un cas de pneumo-entérite.

Solmonella kottbus.

\section{LAPINS}

La seule identification fut réalisée à partir d'une hémoculture provenant d'un lapin de notre élevage :

Solmonella hull.

\section{COBAYES}

De même, le cobaye d'où fut isolée une souche de Salmonella, appartenait à notre élevage :

Solmonello stanleyville.

\section{OISEAUX}

Les déterminations ont été le résultat d'examens bactériologiques effectués sur :

1. - Des cadavres de poules d'élevage reçus par notre service de diagnostics :

Salmonella gallinarum. 26

Salmonella hull ....................... 2

Salmonella onotum ...................... 1

Salmonella mission ..................... 1

Salmonella virchow. ................... 1

Salmonella stanleyville................ 1

Solmonella schwarzengrund............. 1

2. - Sur des cadavres de canards sauvages abattus par des chasseurs (Dendrocygna fulva). Sur 6 examens effectués, une salmonelle fut isolée.

Salmonello hull.

\section{REPTILES}

Incidemment, nous avons été amenés à isoler une Shigella sonnei dans les excréments d'un varan (Varanus exanthematicus). Ce fait intéressant, nous a incité à rechercher systématiquement les shigelles et salmonelles dans les excréments de varans.
Sur 25 varans (Varanus exanthematicus ef Varanus niloticus) examinés, nous avons rencontré 15 porteurs de salmonelles et isolé 9 souches différentes :

Solmonella amager ...................., 2

Salmonello onotum ....................... 1

Solmonella ardwick..................... 1

Salmonella cubana ................... 3

Salmonella langerhorn .................. 1

Solmonella conostel ..................... 1

Salmonella hull ......................, 1

Solmonella riggil (nouveau sérotype)....... 3

Salmonello massokory (nouveau sérotype)..., 2

\section{BATRACIENS}

Des crapauds (espèce indéterminée) sont souvent rencontrés dans les réservoirs d'eau destinée à la consommation humaine; nous avons voulu préciser la possibilité de contamination salmonellique. Sur 25 crapauds examinés, 13 souches de Salmonelles ont été isolées dont 8 différentes :

Salmonella colombo................... 1

Solmonella hull ...................... 2

Solmonella rubislow .................. 4

Solmonello stanleyville .................. 1

Solmonella thioroye..................... 1

Solmonello djermaï (nouveau sérotype)...... 1

Salmonella dougi (nouveau sérotype)......... 1

Salmonella goulfey (nouveau sérotype)....... 2

\section{EAU}

A la suite d'un examen bactériologique effectué à la demande de la Régie des Eaux de Fort-Lamy, a été isolée :

Solmonello give.

Les tableaux ci-joints réunissent les identifications par espèce et précisent les sérotypes de chaque Salmonelle.

\section{DISCUSSION}

De notre enquête épidémiologique, il semble que l'on puisse déduire les faits suivants :

1. - La grande importance de la contamination humaine surtout pendant la saison des pluies 


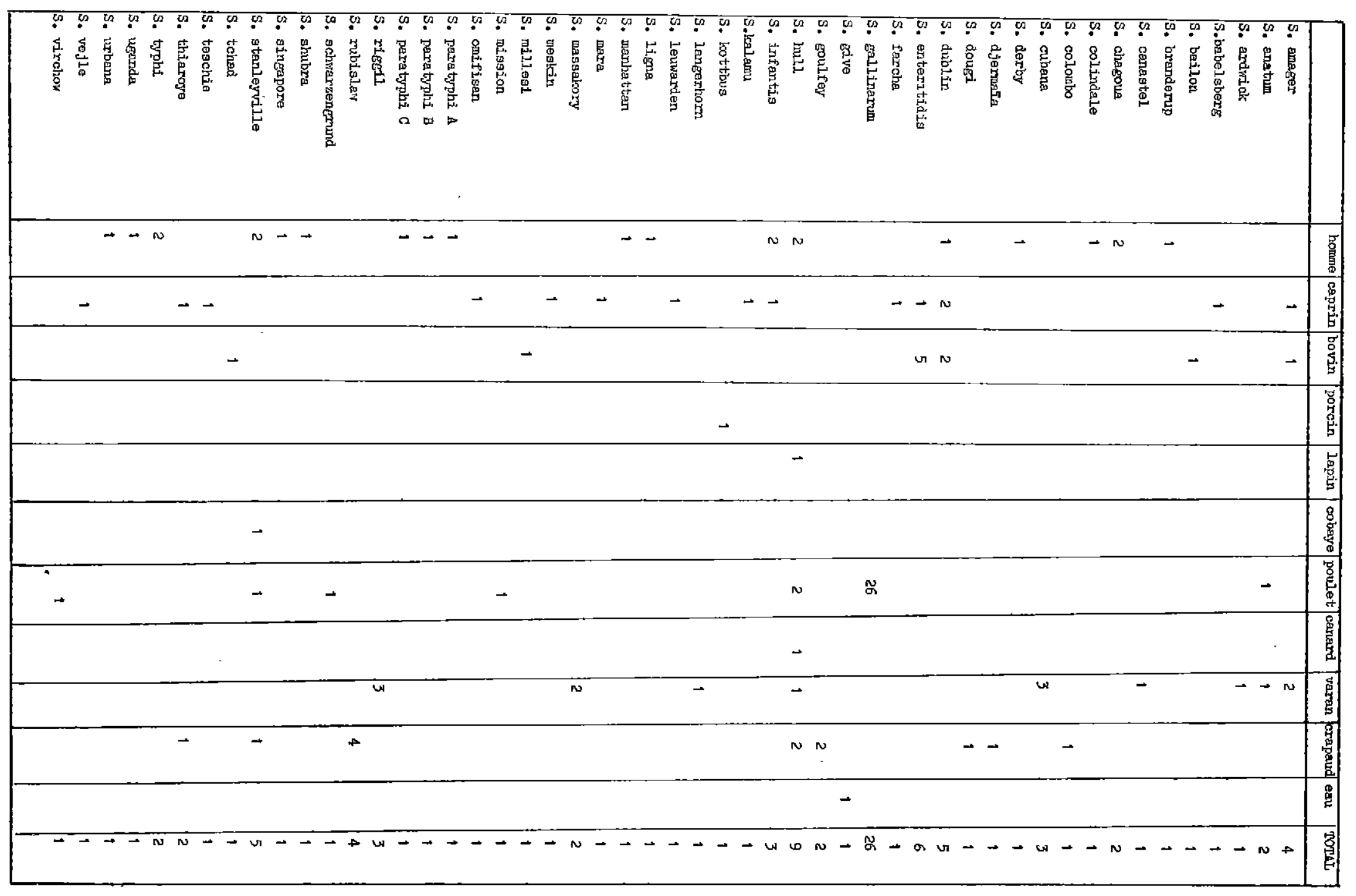


NOMS

1. S. amager

2. S. anatum

3. S. ardwick

4. S. babelsberg

5. S. bailon

6. S. branderup

7. S. canasiel

8. S. chagoua

9. S. colindale

10. S. colombo

11. S. cubana

12. S. derby

13. S. djermaia

14. S. dougi

15. S. dublin

16. S. enteritidis

17. S. farcha

18. S. gallinarum

19. S. give

20. S. goulfey

21. S. hull

22. S. infantis

23. S. kalamu

24. S. kottbus

25. S. langerhorn

26. S. leuwarden

27. S. ligna

28. S. manhattan

29. S. mara

30. S. massakory

31. S. meskin

32. S. millesi

33. S. mission

34. S. omifisan

35. 5. paratyphi A

36. S. paratyphi B java

37. S. paratyphi C

38. S, riggil

39. 5. rubislaw

40. S. schwarzengrund

41. S. shubra

42. S. singapore

43. 5. stanleyville

44. S. tchad

45. S. teschie

46. S. typhi

47. S. uganda

48. S. urbana

49. S. vejle

50. S. virchow
SEROTYPES

$3,10: y: 1,2$

$3,10:$ eh $: 1,7$

$6.7: f_{1} g:-$

$28 z_{4} ; Z_{23}:$ en $z_{15}$

$9,46: 0: e, n_{1} x, z_{16}$

$6,7:$ eh : e, $n, z_{15}$

$9,12: z_{20}: 1,5$

$1,13,23,37: a: 1,5$

$6,7: r: 1,7$

$38: y: 1,6$

$1,13,23: z_{20}:-$

U, 4, 12:fg:-

$28: z_{29}$

$50: y: 1,6$

$1,9,12: 9:-$

$1,9,12: \mathrm{gm} \cdot$

$43: y: 1,2$

$1,9,12:-$

$3,10:$ Iv $: 1,7$

$1,40: k: 1,5$

$16: b: 1,2$

$6,7:$ e : $1,5^{\circ}$

4, $12: z_{4} z_{24}:-$

$6,8:$ eh, $: 1,5$

$18: \mathrm{mt}$

$11: b: 1-5$

$35: z_{10}: z_{6}$

$6,8: d: 1,6$

39 : eh:- :

$35: r:$ lw.

51 : eh $: 1,2$

$40:$ Iv $: 1,2$

$6,7: d: 1,5$

$40: z_{29}$

$1,2,12: a:-$

$1,4,5,12: b: 1,2$

6,7 vi $: c 1,5$

6,7 : gt:-

$11: r: e, n, x$

$1,4,12,27: d: 1,7$

$4,5,12,: z: 1,2$

$6,7: k: e, n_{1} x$

$4,5,12: z_{4}, z_{28}:(1,2)$

$35: b:-$ :

$47: z_{13}, z_{18},: e, n, z_{15}$

9,12, vi $: d:-$

$3,10: l z_{13}: 1,5$

$30: b: e, n, x$.

$3,10:$ eh $: 1,2$

$6,7: r: 1,2$
HOTES

caprins, bovins, varans

poulet, varan

varan

caprin

bovin

homme

varan

homme (3)

homme

cropaud

varan

homme

crapaud (4)

crapoud (4)

homme, coprin, bovin

caprin, bovin

caprin (1)

poulet

eau

crapaud (4)

homme Iapın, poulet,

varan, crapaud, canard

homme caprin

caprin

porcin

varan

caprin

homme (3)

hornme

caprin, varan (3).

varan (3)

caprin (3)

bovin (2)

poulet

caprin

homme

homme

homme

varan (3)

crapaud

poulet

homme

homme

homme, cobaye, poulel

crapoud

caprin (2)

caprin

homme

homme

homme

caprin

poulet 
et la grande diversité des sérotypes. Dix-sept différents ont été isolés.

2. - La rareté des salmonelles dans les ganglions mésentériques de bovins, caprins, porcins, «animaux sains», abattus pour la consommation humaine.

Sur 272 bovins examinés, 4 souches isolées Sur 43 caprins examinés, 0 souche isolée

Sur 17 porcins examinés, 0 souche isolée

3. - La plus grande fréquence de Salmonelies isolées dans les ganglions mésentérıques de caprins inoculés de virus capripestique.

Sur 120 animaux examinés, 14 souches de salmonelles ont été isolées.

4. - La très grande contamination des crapauds (52 pour 100) et des varans (Varonus exanthematicus et niloticus) (60 pour 100). II semble même que la contamination de Varanus exanthematicus soit encore plus importante.

5. - La diversité des Salmonelles isolées chez les poulets morts avec des signes cliniques de typhose :

Salmonella gallinarum.............. 26

Salmonella onotum. ..................... 1

Salmonella hull...................... 2

Salmonella mission.................... 1

Salmonella schworzengrund............... 1

Salmonella stanleyville.................. 1

Salmonella virchow. ...................., 1

II ressort que 20 pour 100 des cas du syndrome typhose semblent être dus d̀ des sérotypes différents de Salmonella gallinarum.

6. - L'ubiquité des différents sérotypes qui offre un intérêt épidémiologique certain (voir tableau 1).

\section{Exemple:}

Salmonella hull a été rencontrée :

chez l'homme................... 2 fois

chez le lapin.................. 1 fois

chez le poulet................. 2 fois

chez le canard.................., 1 fois

chez le varan................... 1 fois

chez le crapaud.................. 2 fols

Salmonella stanleyville a été rencontrée:

chez !'homme.................. 2 fois

chez le cobaye.................., 1 fois

chez le poulet.................. 1 fois

chez le crapaud .................. 1 fois

Solmonella dublin :

chezl'homme.................., 1 fois

chez les bovins................., 2 fois

chez les caprins............... 2 fois

\section{Samonello enteridis :}

chez les caprins.................. 1 fois

chez les bovins................ 5 fois

\section{Samonella infontis :}

chez l'homme................... 2 fois

chez les coprins................. 1 fois

\section{Salmonella amager :}

chez les coprins...................., 1 fois

chez le varan................... 2 fois

\section{Samonello anatum :}

chez le poulet.................. 1 fois

chez le varan. ................. 1 fois

\section{Samonella thioroye :}

chez les caprins................... 1 fois

chez les crapauds..............., 1 fois

\section{SUMMARY}

The different serotypes of salmonella identified in the Republic of Chad.

Routine diagnostic and systematic investigation carried out by the laboratory of Farcha in the Republic of Chad during the past ten years has resulted in the isolation, in man and in nine species of animal, of one hundred and sixteen strains of salmonella, beionging to fifty serotypes, of which twelve are new. 


\section{RESUMEN}

Diferentes tipos de saimonela aislados en la Republica del Chad.

Los diagnosticos rutinarios las encuestas sistematicas efectuadas por el laboratorio de Farcha en la republica del Chad, desde hace deiz anos, han permitido el aislamiento en el hombre $y$ en nueve especias animales, de ciento dieciseis cepas de salmonelas; perteneciendo a cincuenta serotipos diferentes de los cuales doce son nuevos.

\section{BIBLIOGRAPHIE}

1. LE MINOR (L.), THOME (M.), PERREAU (P.) etCHARIE-MARSAINES (Ch.), - «Un nouveau sérotype de Salmonella : Salmonella Farcho. » Ann. Inst. Past., 1959, 97 (1) : 107-108.

2. LE MINOR (L.), THOME (M.), PERREAU (P.) et CHARIE-MARSAINES (Ch.). - Deux nouveaux sérotypes de Salmonella : Salmonella millesi $(40:$ IV 1,2) et Salmonella Tchad (35: b : -). Ann. Inst. Pasteur ; 1959, 97 (3), 406-407.

3. LE MINOR (L.), VIGIER (M.), THOME (M.), CHARIE-MARSAINES (Ch.) et PERREAU (P.). - Ann. Inst. Posteur, 1963, 104 : 830-832.
4. LE MINOR (L.), CHARIE-MARSAINES (Ch.), ZAJC-SATLER (J.) ef DELAGE (R.), BORIES (S.), PERPEZAT (A.), ET SEGONNE (J.).« Nouveaux sérotypes de Salmonella identifiés. » Ann. Inst. Posteur, 1963, 106.

5. LE MINOR. - Le diagnostic de laboratoire des entérobactéries. Editions de la Tourelie 1963.

6. PLOWRIGHT (W.). - A note on Salmonella infection of adult cattle in plateau province Nigeria. Bull. épiz. Dis. ofr. 1957, $5: 337$ 341. 